\title{
Triagem Auditiva Neonatal: Experiências de Diferentes Países
}

\section{Newborn Hearing Screening: Experiences of Different Countries}

\author{
Fernanda Soares Aurélio*, Tania Maria Tochetto**.
}

\begin{abstract}
* Mestre em Distúrbios da Comunicação Humana pela Universidade Federal de Santa Maria - UFSM. Fonoaudióloga
** Doutora em Distúrbios da Comunicação Humana pela Universidade Federal de São Paulo - UNIFESP. Professor Associado da Universidade Federal de Santa Maria no Curso de Fonoaudiologia e Programa de Pós-Graduação em Distúrbios da Comunicação Humana.

Instituição: Universidade Federal de Santa Maria - UFSM. Santa Maria / RS - Brasil.

Endereço para correspondência: Fernanda Soares Aurélio - Rua Gonçalves Chaves, 812 - Apto. 301 -Centro - Pelotas / RS - Brasil - CEP: $96015-560$ - Telefone: (+55 55) 9159-8852; (+55 53) 3025-7220 - E-mail: fernandaurelio@yahoo.com.br Artigo recebido em 3 de Março de 2009. Artigo aprovado em 19 de Abril de 2009.
\end{abstract}

\section{RESUMO}

Introdução:

Objetivo:

Método:

Síntese dos dados:

Conclusão:

Palavras-chave:

\section{SUMMARY}

Introduction:

Objective:

Method:

Data Synthesis:

Conclusion:

Keywords:
A Triagem Auditiva Neonatal (TAN) tem sido implementada em diversos países.

O propósito deste estudo foi conhecer e relatar as experiências de diferentes países com a TAN. Realizou-se busca nas bases de dados MEDLINE, BIREME, SCIELO, LILACS, PUBMED, GOOGLE SCHOLAR, Science Direct, periódicos CAPES e nos sites do Grupo de Apoio a Triagem Auditiva Neonatal Universal (GATANU), Joint Committee on Infant Hearing, International Association of Logopedics and Phoniatrics e American Academy of Pediatrics (AAP), além da pesquisa em anais de eventos científicos e livros texto, quando necessário. Os descritores usados foram: triagem auditiva neonatal, triagem auditiva neonatal universal, newborn hearing screening e universal newborn hearing screening. Constatou-se que os programas de TAN vêm sendo realizados em aproximadamente 55 países com eficácia e sucesso. O procedimento predominante é a avaliação de Emissões Otoacústicas Evocadas (EOAEs).

São crescentes os esforços pela melhoria da qualidade dos programas, universalização da TAN e elaboração de legislação pertinente.

triagem neonatal, recém-nascido, perda auditiva.

The Newborn Hearing Screening (NHS) has been implemented in several countries.

The purpose of this study was to identify and report the experiences of different countries with the NHS.

We searched the MEDLINE, BIREME, SciELO, LILACS, PubMed, Google Scholar, Science Direct, CAPES journals, and the websites of the Support Group Universal Newborn Hearing Screening (GATANU), Joint Committee on Infant Hearing, International Association of Logopedics and Phoniatrics, American Academy of Pediatrics (AAP), and research in scientific events and textbooks as needed. The keywords used were: newborn hearing screening, universal newborn hearing screening, newborn hearing screening, and universal newborn hearing screening.

We found that TAN programs are being conducted in approximately 55 countries effectively and successfully. The predominant procedure is the assessment of evoked otoacoustic emissions (SOAE). There are increasing efforts to improve the quality of programs, universal TAN and development of relevant legislation.

newborn screening, newborn, hearing loss. 


\section{INTRODUÇÃO}

O diagnóstico precoce da perda auditiva direciona o planejamento e a introdução de medidas terapêuticas, objetivando a prevenção de agravos e melhoria da qualidade de vida da criança e da família.

A Triagem Auditiva Neonatal (TAN) tem por objetivo separar com acurácia e eficiência a grande maioria dos neonatos com boa audição, daqueles com perda auditiva.

A difusão das consequências da perda auditiva sobre o desenvolvimento social, emocional, cognitivo e linguístico resultou na conscientização dos governos de vários países que implantaram programas de TAN.

Em diversos países, a TAN vem sendo implementada e, apesar das barreiras encontradas, continua sendo realizada e aperfeiçoada de acordo com as possibilidades de cada local. A literatura registra a viabilidade da TAN e sua eficácia na detecção precoce da deficiência auditiva infantil.

O propósito deste estudo foi conhecer e relatar as experiências de diversos países com a TAN.

\section{REVISÃO DE LITERATURA}

A implantação e a manutenção de programas de TAN têm despertado o interesse e preocupação de Fonoaudiólogos, Otorrinolaringologistas e Pediatras, dada a relevância de garantir o diagnóstico e a intervenção precoces da deficiência auditiva (1).

Nos países em desenvolvimento, os Programas de Triagem Auditiva Neonatal (PTAN) vêm sendo realizados desde 1986, sendo a Índia o país pioneiro (em neonatos de risco). Omã foi o primeiro país com PTAN de âmbito nacional e no Irã existem estudos piloto em 28 de 30 províncias. Em Singapura a TAN tem sido executada em clínicas de imunização. O procedimento foi recusado por $59 \%$ das famílias de crianças de quatro meses ou menos (2).

Estudo de Masoud et al. (2006) relata que dos países em desenvolvimento o Brasil é o que possui maior número de serviços que disponibilizam a TAN (237 locais) (3). Cento e trinta e sete instituições, entre maternidades e clínicas, estão cadastradas no Grupo de Apoio a Triagem Auditiva Neonatal Universal (GATANU). A região sudeste, formada pelos estados de São Paulo, Rio de Janeiro, Espírito Santo e Minas Gerias, é a que apresenta maior número de programas de TAN (63 serviços), seguida da região sul composta pelos estados do Rio Grande do Sul, Santa Catarina e Paraná (34 serviços). A região norte (Acre,
Amapá, Amazonas, Rondônia, Roraima, Pará e Tocantins)é a que apresenta o menor número de programas cadastrados no GATANU, sendo quatro em maternidades e um em clínica particular (4).

No Brasil, os primeiros programas de TAN foram implantados em 1987, um no Hospital São Paulo (São Paulo, SP) outro no Hospital Universitário de Santa Maria (Santa Maria, RS). O procedimento utilizado em ambas as instituições foi a observação de respostas comportamentais (5).

No ano seguinte, no Hospital Israelita Albert Einstein, teve início o primeiro programa de TAN que utilizou método eletrofisiológico (Potencial Auditivo de Tronco Encefálico - PEATE) e contou com fonoaudiólogo na equipe neonatal. Inicialmente abrangia apenas os neonatos com indicadores de risco para surdez, mas gradualmente foi ampliado para todas as crianças da Unidade de Terapia Intensiva Neonatal (UTIN). A partir de 1999 a avaliação de Emissões Otoacústicas Evocadas (EOAEs) passou a ser utilizada (6).

Na Europa, os programas de TAN envolvem todo o continente, e atualmente, cobrem mais de 90\% da Áustria, Bélgica (parte flamenga), Dinamarca, Croácia, Inglaterra, Luxemburgo, Países Baixos e Polônia. Uma implementação parcial tem sido feita na Alemanha, Itália, Lituânia, Malta, Espanha, Suécia, Suíça e País de Gales. Já a parte francesa da Bélgica, Chipre, França e Irlanda estão em fase mais avançada, enquanto a República Tcheca, Estônia, Finlândia, Grécia, Hungria, Letônia, Noruega, Portugal, Romênia, Escócia, Eslováquia, Eslovênia e Turquia encontram-se em fase de projeto piloto (7).

Os PTAN da Inglaterra triam cerca de 1700 crianças a cada dia e são reconhecidos como os mais avançados em todo o mundo. Mais de 3400 crianças com deficiência auditiva já foram identificadas (8).

Ao estabelecer padrões de qualidade para os programas de TAN a American Academy of Pediatrics (AAP, 1999) (9) sugeriu que os mesmos tivessem caráter universal, índice de falso-positivo inferior ou igual a 3\%, que os encaminhamentos para a etapa de diagnóstico não excedessem $4 \%$ e os procedimentos utilizados fossem EOAEs e/ou o PEATE. Ainda, a TAN deveria ser realizada antes da alta hospitalar, entre as primeiras $24 \mathrm{e} 48$ horas de vida. Em caso de falha, o reteste deveria ser realizado dentro de um mês. O diagnóstico deveria ser concluído antes dos três meses de vida e a intervenção iniciada antes dos seis. Os programas de TAN devem ser avaliados de acordo com as normas da AAP (1999).

A TAN tem caráter universal quando são triados no mínimo 95\% dos neonatos (AAP, 1999). Há relatos de 
Tabela I. Instituições, municípios/estados ou localidades e países que empregam somente EOAEs na execução da TAN.

\begin{tabular}{lll}
\hline País & $\begin{array}{l}\text { Munićíio/Estado } \\
\text { ouLocalidade }\end{array}$ & Instituição \\
\hline África do Sul (29) & Petrória/Gauteng & NC \\
Alemanha( I2) & Hamburgo & Marien Hospital \\
Arábia Saudita(25) & NC & NC \\
Áustria (30) & NC & NC \\
Brasil (24) & São Paulo / SP & Hospital IsraelitaAlbertEinstein \\
Eslováquia(26) & Limbova & Children's UniversityHospital \\
Espanha (31) & Gijón/Astúrias & Hospital de Cabueñes \\
Filipinas (32) & Manila & Hospital Geral das Filipinas \\
Grécia (33) & Atenas & Hospital Hippokration \\
Itália (34) & Roma & NC \\
Iran(3) & Tehran, Mashad & NC \\
Paquistão (35) & Lahore & NC \\
Polônia & Poznan (36) & Hospital da Universidade de Poznan \\
& Zabrze (37) & SilesianMedicalAcademy \\
Qatar (38) & Doha & NC \\
\hline
\end{tabular}

Tabela 2. Instituições, municípais/estados ou localidades e países que utilizam EOAEs na TAN e, em caso de falha ou em RN's com indicadores de risco para a deficiência auditiva, avaliam PEATE para o diagnóstico.

\begin{tabular}{lll}
\hline País & $\begin{array}{l}\text { Município/Estado } \\
\text { ouLocalidade }\end{array}$ & Instituição \\
\hline Espanha & Cantábria(I I) & NC \\
& Valladolid (39) & Hospital Universitário de Valladolid \\
França ( I 6) & Eure & Departamento Geográfico de Eure \\
Israel (40) & NC & NC \\
Itália (4 I) & Milão & NC* \\
Jordânia (42) & NC & NC \\
Malásia (42) & Kuala Lumpur & Hospital Universitário de Kebangsaan Malásia \\
Taiwan (43) & NC & Mackay Memorial Hospital \\
\hline
\end{tabular}

* Instituição que implementou a TAN; U.O. Neurologia-Neurofisiopatologia and Dipartimento di Neonatologia of de Istituti Clinici di Perfezionamento.

EOAEs: Emissões Otoacústicas Evocadas; TAN: Triagem Auditiva Neonatal; RNs: recémnascido; PEATE: Potencial Evocado Auditivo de Tronco Encefálico; NC: Não consta a informação no estudo citado.

Triagem Auditiva Neonatal Universal (TANU) no México (Monterrey) (10), na Espanha (Cantábria) (11), na Alemanha (Hamburgo) (12), Estados Unidos (EUA) (estados com legislação implementada (13), estado do Mississipi (14), estado do Novo México (15); França (Eure) (16); Noruega (Condado de Ostfold) (17); nos hospitais públicos de Singapura (2), na Austrália ocidental (Perth) (18), Nigéria (19, 20), África do Sul (21) e Hong Kong (22).

O índice aceitável de resultados falso-positivo conforme a AAP (1999) é igual ou inferior a 3\%. Os países que satisfizeram tal critério foram México (10) e EUA (23).
O índice de encaminhamento para diagnóstico foi inferior a 4\% (AAP, 1999) no Brasil (24), na Noruega (Condado de Ostfold) (17), Arábia Saudita (25), Nigéria (20), Eslováquia (Limbova) (26), Oman (27), Singapura (28) e México (10).

Os procedimentos indicados pela AAP (1999) são EOAEs e/ou PEATE. Os mais comumente empregados nos programas de TAN nos diferentes países são apresentados nas Tabelas 1, 2, 3 e 4.

Além dos procedimentos referidos nas Tabelas 
Tabela 3. Instituições, municípios/estados ou localidades e países que utilizam EOAEs na TAN, e, em caso de falha, AABR.

\begin{tabular}{lll}
\hline País & $\begin{array}{l}\text { Munićípio/Estado } \\
\text { ouLocalidade }\end{array}$ & Instituição \\
\hline Inglaterra(44) & NC & NC \\
Noruega(17) & Condado de Ostfold & NC \\
Oman (27) & NC & NC \\
\hline
\end{tabular}

EOAEs: Emissões Otoacústicas Evocadas; TAN: Triagem Auditiva Neonatal; AABR: Automated Auditory Brainstem Response (Resposta Auditiva Automática de Tronco Encefálico); NC: Não consta a informação no estudo citado.
Tabela 4. Instituições, municípios/estados ou localidades e países que utilizam EOAEs e/ou AABR na execução da TAN.

\begin{tabular}{lll}
\hline País & $\begin{array}{l}\text { Município/Estado } \\
\text { ouLocalidade }\end{array}$ & Instituição \\
\hline China (45) & Shanghai, Bïjing, Shandong & NC \\
Hong-Kong(46) & Hong-Kong & NC \\
Nigéria $(19,20)$ & Lagos & NC \\
Singapura(2) & Singapura & NC \\
\hline
\end{tabular}

EOAEs: Emissões Otoacústicas Evocadas; TAN: Triagem Auditiva Neonatal; AABR: Automated Auditory Brainstem Response (Resposta Auditiva Automática de Tronco Encefálico); NC: Não consta a informação no estudo citado.

Tabela 5. Países/Instituições que mencionaram a idade na qual a TAN foi realizada.

\begin{tabular}{ll}
\hline País/Instituição & Idade na realização da Triagem Auditiva \\
\hline Brasil/Hospital IsraelitaAlbert Einstein (24) & Segundo ou terceiro dia de vida ou antes da alta, para RN internado em UTIN. \\
Espanha/Hospital de Cabueñes (3 I) & Idade mediana de 7I dias \\
Espanha/Hospital Universitário de Valladolid (39) & Menos de seis meses \\
Grécia / NC (33) & Mis de 36 semanas \\
Itália /NC & 36 horas após o nascimento (Milão) (4I) \\
Itália/NC & Segundo ou terceiro dia de vida (Roma) (34) \\
Nigéria / NC (20) & Idade média de 2.6 meses \\
Noruega / NC( ( 7) & Segundo dia de permanência no berçario \\
Polônia/Hospital de Universidade de Poznan(36) & Segundo outerceiro dia de vida \\
Taiwan/MackayMemorial Hospital(43) & Idade média de 52 horas. \\
\hline
\end{tabular}

RN: Recém-nascido; UTIN: Unidade de Terapia Intensiva Neonatal; NC: Não consta a informação no estudo citado.

1, 2, 3 e 4, no México (Monterrey) (10) a TAN foi realizada utilizando a Resposta Auditiva Automática de Tronco Encefálico (Automated Auditory Brainstem Response - AABR) e em caso de falha foi realizado o PEATE. Na África do Sul (21) foi empregado as EOAEPDs associado à timpanometria de altas frequências e em Limbova (Eslováquia) (47) foi utilizada a pesquisa das EOAETs e, em caso de falha, a realização da timpanometria. Na Noruega (Oslo) (48) e na Austrália (Perth) (18) o uso de EOAEs foi associado à AABR em todas as etapas da triagem. Nos estados do Mississipi (14) e Carolina do Norte (EUA) (23) a triagem foi realizada somente com PEATE. Em um estudo realizado de março de 2000 a dezembro de 2002 em Taiwan (49) os recém-nascidos foram triados com EOAEs associada à PEATE.

Conforme a AAP (1999), a TAN deve ser realizada entre as primeiras 24 e 48 horas de vida. A Tabela 5 apresenta as idades nas quais a TAN é realizada em diferentes países.

No Brasil (24), Itália (Roma (34) e Milão (41)) e Polônia (36) (Hospital da Universidade de Poznan) todas as crianças foram triadas no período recomendado.
A AAP (1999) refere, ainda, que a TAN deve ser realizada antes da alta hospitalar. Das publicações que forneceram tal informação, apenas na Malásia (Hospital Universiti Kebangsaan Malaysia) (42) e na África do Sul (Gauteng) (29) a TAN não seguiu esta norma da AAP.

Em caso de falha na TAN, o reteste deve ser realizado dentro de um mês após a primeira triagem (AAP, 1999). Tal conduta foi adotada no Brasil (São Paulo) (24), na Itália (Milão) (41) e na França (Eure) (16). Na Malásia (Hospital Universiti Kebangsaan Malaysia) (42) as crianças foram retestadas aos dois e, em caso de nova falha, aos três meses, e na África do Sul (Gauteng) (29) o reteste foi realizado seis semanas após a falha na primeira triagem auditiva.

Publicações de dez países citaram a época da conclusão da etapa de diagnóstico: EUA (Colorado $(50,51)$ Mississipi (14)), Espanha (Cantábria) (11), Itália (Sicília) (52), Áustria $(30,53)$, Alemanha $(7,54)$, Singapura (2), Arábia Saudita (25), Nigéria (20), África do Sul (55) e México (Monterrey) (10). O diagnóstico foi realizado no período recomendado em 100\% dos casos apenas em Monterrey (México) (10). No estado do Colorado (EUA) (51), de 1992 à 1999 o diagnóstico foi concluído antes dos 3 meses em 
$71 \%$ dos recém-nascidos triados. Na Nigéria (20) a idade do diagnóstico variou de 46 a 360 dias. Nos demais países a idade média do diagnóstico oscilou entre 3.9 meses (Mississipi/ EUA) (14) e 39 meses (Alemanha) (54).

Nos EUA (50), na Áustria (53) e na Alemanha (54) a idade da identificação da deficiência auditiva nas crianças não triadas foi superior à daquelas que realizaram a TAN. No Colorado (EUA) (50) o diagnóstico foi realizado, embora tardiamente, antes dos seis meses em $84 \%$ das crianças submetidas a TAN e em apenas $8 \%$ das crianças não triadas. $\mathrm{Na}$ Áustria (53) e na Alemanha (54) as crianças que não realizaram a TAN tiveram a deficiência auditiva diagnosticada em média aos 37.6 e 17.8 meses respectivamente. Já as crianças triadas tiveram o diagnóstico concluído aos 3.9 (Áustria (53)) e 3.1 meses de idade (Alemanha (54)).

Segundo a AAP (1999) e o Joint Committee on Infant Hearing (JCIH) (2007) (56) a intervenção deve ser iniciada antes dos seis meses de idade. Das publicações que referiram a época em que foi feita a intervenção, a conduta sugerida foi adotada somente na Itália (Sicília) (52), e na a Áustria (53) nas crianças que realizaram a TAN. Em outra publicação desse mesmo país (30), de 1990 a 2006, 61\% das crianças triadas foram submetidas à intervenção adequada até os seis meses de idade, contra somente $4 \%$ das crianças que não realizaram a TAN. Na Alemanha (54) a idade das crianças triadas no momento da intervenção teve mediana de 3.5 meses e em crianças não triadas a idade no diagnóstico teve mediana de 21 meses.

Na Espanha (Cantábria) (11) apenas 50\% das crianças começaram o tratamento antes dos seis meses. No México (Monterrey) (10), apesar de 100\% dos diagnósticos serem realizados no período indicado, todas as crianças diagnosticadas tiveram intervenção após os seis meses de idade. Em Cuba (57) a intervenção iniciou em média aos 10 meses de idade, em Singapura (2) aos 42.4 meses e no estado do Mississipi (EUA) (14) aos 6.1 meses.

No Brasil há Leis tornando a TAN obrigatória nos Estados do Paraná, Pernambuco e São Paulo (5), Santa Catarina, Minas Gerais, Piauí, Rondônia e Mato Grosso do Sul (58). O Projeto de Lei (de âmbito federal) no 697/07 (em tramitação), dispõe sobre a obrigatoriedade do exame "Emissões Otoacústicas Evocadas - EOA", conhecido como "teste da orelhinha" para todos os recém nascidos no País (58).

Na Alemanha, há esforço legislativo para implantar a TANU como procedimento regular oferecido a todos os recém-nascidos (7).

Nos Estados Unidos (EUA) os estados onde a TANé obrigatória por força de lei, segundo a Centers for Disease
Control and Prevention (2007) (59), são: Arkansas, Califórnia, Colorado, Connecticut, Florida, Geórgia, Hawaí, Illinois, Indiana, Iowa, Kansas, Kentucky, Louisiana, Maine, Maryland, Massachusetts, Mississippi, Missouri, Montana, Nebraska, Nevada, New Hampshire, New Jersey. New York, North Carolina, Ohio, Oklahoma, Oregon, Pennsylvania, Rhode Island, South Carolina, Texas, Utah, Virginia, West Virginia, Wisconsin e Wyomin.

Conforme mencionado por Green et al. (2007) (13), nos EUA o índice de realização da TAN nos estados que dispõem de legislação é mais elevado do que nos demais. Os estados que triaram $95 \%$ dos neonatos eram, em sua maioria, aqueles com legislação implementada.

\section{DISCUSSÃO}

É crescente o número de países conscientes da necessidade da execução de programas de TAN. Tais programas aumentam significativamente o índice de diagnóstico precoce da deficiência auditiva infantil e vêm sendo executados de maneira eficiente e eficaz em diversos locais.

Programas de TAN vêm sendo implementados como parte dos sistemas de saúde em aproximadamente 55 países, onde a busca por melhorias é crescente. Nos países onde a TAN ainda não é executada, há um árduo esforço para a implementação da mesma.

A implementação de programas de TAN tanto em países desenvolvidos quanto nos em desenvolvimento esbarra em obstáculos, tais como: falta de ambiente adequado para a realização dos testes, poucos profissionais competentes e preparados para tal tarefa, escassez de profissionais que realizem a TAN nos finais de semana, carência de serviços para seguimento e controle e, principalmente, pouca informação acerca dos benefícios proporcionados à criança surda (60), tanto por parte dos profissionais quanto da população em geral. Esta situação dificulta o processo de universalização da TAN e acarreta elevado número de desistências antes da realização de todas as etapas necessárias (44).

Faz-se necessária a realização de programas de conscientização sobre a importância da Triagem Auditiva Neonatal e dos benefícios proporcionados por este programa.

Uma das principais consequências da falta de legislação em favor da TAN é o diagnóstico tardio da deficiência auditiva infantil e o baixo índice de crianças triadas. A idade da identificação da deficiência auditiva nas crianças não triadas é superior à das crianças que realizaram a TAN (50, 
53, 54). Em decorrência disto, muitos países vêm realizando esforços para aprovar e implementar leis para a execução da TAN e garantir assim, que todas as crianças tenham a sua audição testada em tempo hábil.

O caráter universal e obrigatório da TAN tem sido ressaltado exaustivamente, para que nenhuma criança com deficiência auditiva deixe de ser diagnosticada e receba a intervenção necessária para um adequado desenvolvimento social, psíquico, educacional e linguístico. Mesmo assim pôde-se verificar que, apesar das recomendações da AAP (1999) e do JCIH (2007), há elevado número de programas que praticam a Triagem Auditiva seletiva.

Verificou-se que a TAN vem sendo aplicada, em alguns locais, após o terceiro mês de vida, o que protela o diagnóstico e a intervenção.

Os procedimentos utilizados com maior frequência são comuns aos países desenvolvidos e em desenvolvimento. O procedimento mais utilizado na TAN tem sido a análise de EOAEs. Em caso de falha a conduta mais frequente é o reteste com EOAEs e, somente em caso de nova falha, as crianças são encaminhadas para avaliação de PEATE ou AABR para diagnóstico. O que se mostrou bastante variável nos diferentes estudos foi o tempo estipulado para as crianças que falharam na triagem retornarem para o reteste. Este tempo variou de sete dias (Santa Maria/Brasil) a dois meses (Malásia) (54)

A TAN tem se mostrado um procedimento bastante eficaz e praticável. Sua relevância reside na redução na idade das crianças na época do diagnóstico e da intervenção, especialmente nos locais providos de legislação.

Assim, o caminho parece ser a busca de melhorias nos programas já existentes, a implementação de novos programas e a regulamentação de leis que os amparem.

\section{COMENTÁRIOS FinAIS}

As consequências da perda auditiva e a relevância da TAN ainda são assuntos pouco conhecidos pela população em geral. Portanto, se faz necessário difundir conhecimentos a respeito destas duas temáticas, esperando mudança de comportamento e incorporação da TAN como procedimento de rotina entre profissionais e pais.

Nos últimos 10 anos, período pesquisado para a elaboração desse texto, programas de TAN vêm sendo realizados em aproximadamente 55 países com bastante eficácia e sucesso, tendo como principal procedimento a análise de EOAEs. São crescentes os esforços para a melhoria dos mesmos de acordo com as normas estipula- das pela AAP (1999) e pelo JCIH (2007) e ainda para a diminuição dos obstáculos encontrados e elaboração de legislação específica.

Enfim, faz-se necessária a união de esforços por parte dos profissionais, dos pais e dos governos, para que os programas de TAN possam cumprir seus objetivos em benefício de um número cada vez maior de crianças surdas e suas famílias.

\section{REFERÊNCIAS BIBLIOGRÁFICAS}

1. Pádua FGM, Marone S, Bento RF, Carvallo RMM, Durante AS, Soares JC, Barros JCR, Leon ICR. Triagem Auditiva Neonatal: Um Desafio para sua Implantação. Arq. Otorrinolaringol. 2005, 9(3):190-4.

2. Low WK, Pang KY, Ho LY, Lim SB, Joseph R. Universal newborn hearing screening in Singapore: the need, implementation and challenges. Ann Acad Med Singapore. 2005, 34(4):301-6.

3. Masoud AD, Mohsen F, Behzad M, Roshnanak V, Nikta H, Shahrzad, M. Preliminary report of newborn hearing screening in Iran [abstract]. In Proceedings of NHS2006 Conference: Beyond newborn hearing screening: infant and childhood hearing in science and clinical practice; 200631 May - 3 June; Edited by: Grandori F, Hayes D. Lake Como, Italy; 2006:43.

4. Grupo de Apoio a Triagem Auditiva Neonatal Universal (GATANU) [homepage on the Internet]. Programas de Triagem Auditiva Neonatal. Disponível em: <http:// www.gatanu.org/programas/pais.php?menus $=. . /$ menus/ menutan.php\&programa=tan>. Acessado em: 20 jan 2008 .

5. Tochetto TM, Vieira EP. Legislação brasileira sobre triagem auditiva neonatal. 1a ed. São Paulo: Pro-fono; 2006.

6. Grupo de Apoio a Triagem Auditiva Neonatal Universal (GATANU) [homepage on the Internet]. A trajetória da Triagem Auditiva Neonatal no Brasil. Disponível em: $<$ http:/ /www.gatanu.org/gatanu/trajetoria.php $>$. Acessado em: 20 jan 2008.

7. Neumann, K [homepage on the Internet]. Triagem Auditiva Neonatal Universal - Programas de Detecção e Intervenção Auditiva Precoce (Early Hearing Dectection and Intervention-EHDI) na Europa. Disponível em: http:// www.ialpsp.com.br/brasil/convidados.asp. Acessado em: 20 jan 2008.

8. Zamp Bionews [homepage on the Internet]. NHS Screens Two Millionth Baby For Hearing Problems, UK. Sunday, 
September 16th, 2007. Disponível em: http:// www.zampbioworld.org/bionews/index.php/2007/09/16/ 3871. Acessado em: 10 abr 2008.

9. American Academy of Pediatrics. Task force on newborn and Infant Hearing Loss: Detection and Intervention. Pediatrics. 1999, 103(2):527-30.

10. Yee-Arellano HM, Leal-Garza F, Pauli-Müller K. Universal newborn hearing screening in Mexico: results of the first 2 years. Int J Pediatr Otorhinolaryngol. 2006, 70(11):186370.

11. González ALA, Bonilla MC, Morales AC, Gómez CF, Barrasa BJ. Universal newborn hearing screening in Cantabria (Spain): results of the first two years. An Pediatr (Barc). 2005, 62(2):135-40.

12. Kehrl W, Geidel K, Wilkens LM, Löhler J. Universal newborn hearing screening in Marien Hospital Hamburg from September 1999 till April 2002. Laryngorhinootol. 2003, 82(7):479-85.

13. Green DR, Gaffney M, Devine O, Grosse SD. Determining the effect of newborn hearing screening legislation: an analysis of state hearing screening rates. Public Health Rep. 2007, 122(2):198-205.

14. Connolly JL, Carron JD, Roark SD. Universal newborn hearing screening: are we achieving the Joint Committee on Infant Hearing (JCIH) objectives? Laryngoscope. 2005, 115(2):232-6.

15. Mcdaniel SL, Olguin M, Horn KL... Hearearly: New Mexicos universal newborn hearing screening program. Otolaryngol Clin North Am. 1999, 32(6):987-98.

16. De Barros AB, Lenoir FM, Brami P, Kapella M, Obstoy MF, Amstutz-Montadert I, Lerosey Y. Universal hearing screening: 10,835 newborns tested in maternity wards of the geographical Department of Eure, France. Ann Otolaryngol Chir Cervicofac. 2005, 122(5):223-30.

17. Anderssen SH, Andresen J, Andersen R, Sponheim L. Universal neonatal hearing screening of infants with otoacoustic emissions. Tidsskr Nor Laegeforen. 2002, 122(22):2187-9.

18. Bailey HD, Bower C, Krishnaswamy, J, Coates, HL. Newborn hearing screening in Western Australia. Med J Aust. 2002, 177(4):180-5.

19. Olusanya BO, Okolo AA. Early hearing detection at immunization clinics in developing countries. Int J Pediatr Otorhinolaryngol. 2006, 70(8):1495-8.
20. Olusanya BO, Wirz SL, Luxon LM. Hospital-based universal newborn hearing screening for early detection of permanent congenital hearing loss in Lagos, Nigeria. Int J Pediatr Otorhinolaryngol. 2008, 72(7):991-1001.

21. Swanepoel W, Hugo R, Louw B. Infant hearing screening at immunization clinics in South África. Int J Pediatr Otorhinolaryngol. 2006, 70(7)1241-1249.

22. Ng PK, Hui Y, Lam BCC, Goh WHS, Yeung CY. Feasibility of implementing a universal neonatal hearing screening programme using distortion product otoacoustic emission detection at a university hospital in Hong Kong. Hong Kong Med J. 2004, 10:6-13.

23. Clemens CJ, Davis SA, Bailey AR. The False-Positive in Universal Newborn Hearing Screening. Pediatrics. 2000, 106:1-5.

24. Chapchap MJ, Segre CM. Universal newborn hearing screening and transient evoked otoacoustic emission: new concepts in Brazil. Scand Audiol Suppl. 2001, 53:33-6.

25. Habib HS, Abdelgaffar $\mathrm{H}$. Neonatal hearing screening with transient evoked otoacoustic emissions in Western Saudi Arabia. Int J Pediatr Otorhinolaryngol. 2005, 69(6):839-42.

26. Jakubíková J, Kabátová Z, Závodná M. Identification of hearing loss in newborns by transient otoacoustic emissions. Int J Pediatr Otorhinolaryngol. 2003, 67:15-18.

27. Khandekar R, Khabori M, Mohammed A J, Gupta R. Neonatal screening for hearing impairment - The Oman experience. Int J Pediatr Otorhinolaryngol. 2006, 70(4):66370.

28. Tan PL, Daniel LM, Lim SB, Yeoh A, Hee K, Balakrishnan A. The Universal Newborn Hearing Screen (UNHS): Results of a programme for 44,000 infants in KK Womens and Childrens Hospital (KKWCH), Singapore [abstract]. In Proceedings of NHS2006 Conference: Beyond newborn hearing screening: infant and childhood hearing in science and clinical practice; 200631 May - 3 June; Edited by: Grandori F, Hayes D. Lake Como, Italy; 2006:8.

29. Swanepoel D, Ebrahim S, Joseph A, Friedland PL. Newborn hearing screening in a South African private health care hospital. Int J Pediatr Otorhinolaryngol. 2007, 71(6):881-7.

30. Weichbold V, Nekahm-Heis D, Welzl-Mueller K. Tenyear outcome of newborn hearing screening in Austria. Int J Pediatr Otorhinolaryngol. 2006, 70(2):235-40.

31. Colunga JCM, Méndez JCA, Villarreal JMC, Zapico MJA, Estrada CM, Alvarez MLF, Díez FG. Neonatal hearing loss 
screening: our results three years after starting the program. Acta Otorrinolaringol Esp. 2005, 56(2):55-8.

32. Quintos MR, Isleta PF, Chiong CC, Abes GT. Newborn hearing screening using the evoked otoacoustic emission: The Philippine General Hospital experience. Southeast Asian J Trop Med Public Health. 2003, 34 suppl 3:231-33.

33. Korres S, Nikolopoulos TP, Komkotou V, Balatsouras D, Kandiloros D, Constantinou D, Ferekidis E. Newborn hearing screening: effectiveness, importance of high-risk factors, and characteristics of infants in the neonatal intensive care unit and well-baby nursery. Otol Neurotol. 2005, 26(6):118690.

34. Saurini P, Nola G, Lendvai D. Otoacoustic emissions: a new method for newborn hearing screening. Eur Rev Med Pharmacol Sci. 2004, 8(3):129-33.

35. Ali L, Siddiq S, Khan MA, Maqbool S. A hospital-based universal newborn hearing screening programme using transient evoked otoacoustic emission (TEOAE). Pakistan Pediatric J. 2000, 24:117-125.

36. Wroblewska-Seniuk K, Chojnacka K, Pucher B, Szczapa J, Gadzinowski J, Grzegorowski M. The results of newborn hearing screening by means of transient evoked otoacoustic emissions. Int J Pediatr Otorhinolaryngol. 2005, 69(10):13517.

37. Namyslowski G, Morawski K, Urbaniec N, Lisowska G, Trybalska G, Bazowska G, Oslislo A.. The hearing system in newborns from the Upper Silesia. Assessment of TEOAE depending on selected parameters of delivery disorders. Scand Audiol. 2001, 30 suppl 52:21-4.

38. Bener A, Elhakeem AAM, Abdulhadi K. Is there any association between consanguinity and hearing loss? Int J Pediatr Otorhinolaryngol. 2005, 69:327-33.

39. Martínez R, BenitoJI, Condado MA, Morais D, Fernández CJL. Results of one years application of a universal protocol for the early detection of hearing loss in neonates. Acta Otorrinolaringol Esp. 2003, 54(5):309-15.

40. Attias J, Al-Masri M, Abukader L, Cohen G, Merlov P, Pratt H, Othman-Jebara R, Aber B, Raad F, Noyek A. The prevalence of congenital and early-onset hearing loss in Jordanian and Israeli infants. Int J Audiol. 2006, 45(9):52836.

41. Pastorino G, Sergi P, Mastrangelo M, Ravazzani P, Tognola G, Parazzini M, Mosca F, Pugni L, Grandori F. The Milan Project: a newborn hearing screening programme. Acta Paediatr. 2005, 94(4):458-63.
42. Abdullah A, Hazim MYS, Almyzan A, Jamilah AG, Roslin S, Ann MT, Borhan L, Sani A, Saim L, Boo NY. Newborn hearing screening: experience in a Malaysian hospital. Singapore Med J. 2006, 47:60-4.

43. Lin HC, Shu MT, Chang KC, Bruna SM. A universal newborn hearing screening program in Taiwan. Int J Pediatr Otorhinolaryngol. 2002, 63(3):209-18.

44. Kennedy C, Mccann D. Universal neonatal hearing screening moving from evidence to practice. Arch. Dis. Child. Fetal Neonatal. 2004, 89:378-83.

45. Xu A, Zhang C, Du Z. False positives result in newborn hearing screening [abstract]. In Proceedings of NHS2006 Conference: Beyond newborn hearing screening: infant and childhood hearing in science and clinical practice; 200631 May - 3 June; Edited by: Grandori F, Hayes D. Lake Como, Italy; 2006:122.

46. Lam BCC. Newborn Hearing Screening in Hong Kong. Hong Kong Med J. 2006, 12(3):212-18.

47. Jakubíková, J. Závodná, M. Early diagnosis of hearing loss in newborns and children. International Congress Series. 2003, 1240:173-5.

48. Kaldestad RH, Wingaard L, Hansen TW. Screening for congenital hearing loss - a pilot project. Tidsskr Nor Laegeforen. 2002, 122(22):2190-3.

49. Lin CY, Huang CY, Lin CY, Lin YH, Wu JL. Community-based newborn hearing screening program in Taiwan. Int J Pediatr Otorhinolaryngol. 2004, 68(2):185-9.

50. Yoshinaga-Itano C, Coulter D, Thomson V. Developmental outcomes of children with hearing loss born in Colorado hospitals with and without universal newborn hearing screening programs. Seminars in Neonatology. 2001, 6(6):521-529.

51. Mehl AL, Thomson V. The Colorado Newborn Hearing Screening Project, 1992-1999: On the Threshold of Effective Population-Based Universal Newborn Hearing Screening. Pediatrics. 2002, 109:1-8.

52. Martines F, Porrello M, Ferrara M, Martines M, Martines E. Newborn hearing screening project using transient evoked otoacoustic emissions: Western Sicily experience. Int J Pediatr Otorhinolaryngol. 2007, 71:107-12.

53. Weichbold V, Nekahm-Heis D, Welzl-Müller K. Evaluation of the Austrian Newborn Hearing Screening Program / Zehn Jahre Neugeborenen-Hörscreening in Osterreich. Wien Klin Wochenschr. 2005, 117(18):641-6. 
54. Neumann K, Gross M, Bottcher P, Euler HA, SpormannLagodzinski M, Polzer M. Effectiveness and efficiency of a universal newborn hearing screening in Germany. Folia Phoniatr Logop. 2006, 58(6):440-55.

55. Gopal R, Hugo SR, Louw B. Identification and followup of children with hearing loss in Mauritius. Int J Pediatr Otorhinolaryngol. 2001, 57:99-113.

56. Joint Committee on Infant Hearing. Year 2007 Position Statement: Principles and Guidelines for Early Hearing Detection and Intervention Programs. Pediatrics. 2007, 120(4):898-921.

57. Perez-Abalo MC, Gaya JA, Savio G, Ponce De Leon M, Perera M, Reigosa V. Early detection and intervention of hearing impairment in Cuba: outcome after 20 years. Rev Neurol. 2005, 41(9):556-63.
58. Tochetto TM [homepage on the Internet]. Legislação brasileira: análise crítica das leis brasileiras sobre TAN. Encontro Nacional de Triagem Auditiva Neonatal Universal, 3. 2007. Disponível em: <http://www.gatanu.org/ atualidades/cursos.php>. Acessado em: 12 abr 2008.

59. Centers for Disease Control and Prevention. Early hearing detection \& intervention program. Disponível em: $<$ http:/ /www.cdc.gov/ncbddd/ehdi/statesclearinghouse/ default.htm>. Acessado em: 15 abr 2007.

60. Castaño R. Tamizaje universal auditivo neonatal: una utopía para países en desarrollo. Acta Otorrinolaringol Cir Cabeza Cuello. 2002, 30:19-25. 